\title{
Molecular Cytogenetics: the first impact factor (2.36)
}

\author{
Thomas Liehr ${ }^{1 *}$, Henry Heng ${ }^{2^{*}}$, Yuri Yurov ${ }^{3^{*}}$, Aurelia Meloni-Ehrig ${ }^{4}$ and Ivan lourov ${ }^{3^{*}}$
}

We are pleased to announce that Molecular Cytogenetics has received its first official Impact Factor of 2.36 in June 2013.

We sincerely thank our distinguished international editorial board for their efforts on behalf of the journal, and our publisher, BioMed Central, for their inhouse contribution to the speed and efficiency with which manuscripts are processed. Most important of all, of course, are our authors and reviewers [1], and to them we extend our particular thanks.

Since it was launched in 2008, Molecular Cytogenetics has considered 204 and published 179 articles, giving an acceptance rate of $86 \%$. This exemplifies the journal's inclusive editorial policy to publish manuscripts that are scientifically sound, and not just based on perceived impact. These articles cover a wide range of topics in molecular cytogenetics, including clinical and tumor cytogenetics, interphase architecture and karyotype evolution studies not only in human but also in other vertebrates. The journal is also open for molecular cytogenetic-based studies in any species, including invertebrates as well as plants. A rapid review process ensures that papers are published in a timely manner [1]. Our average time from submission to the first decision is 20 days, even under our stringent peer review process. As an open access journal, all papers are made immediately and freely available and articles are being accessed more than ever, with around 27,000 accesses per month. Table 1 shows the 10 most highly accessed articles published in Molecular Cytogenetics during the journal's 5 years of publishing and Table 2 shows

\footnotetext{
* Correspondence: thomas.Liehr@med.uni-jena.de; hheng@med.wayne.edu; y_yurov@yahoo.com; ivan.iourov@gmail.com

${ }^{1}$ Jena University Hospital, Friedrich Schiller University, Institute of Human Genetics, Jena, Germany

${ }^{2}$ Wayne State University School of Medicine, 540 E Canfield Street, Detroit, MI 48201, US

${ }^{3}$ National Research Center of Mental Health, Russian Academy of Medical

Sciences, Moscow, Russia

Full list of author information is available at the end of the article
}

some additional highly cited articles. Authors can also access statistics about their article, such as how many times it has been accessed and discussed on social media websites. These statistics can be viewed by selecting 'Article metrics' in the right hand column of each article. In addition, all Molecular Cytogenetics articles are mobile-device optimized to facilitate easy reading on phones and tablets.

Recently an obituary of a key person in our field, Prof. Dr. Lore Zech, inventor of banding cytogenetics was published and highly recognized in the community [19]. To reflect the needs of our community, in addition to publishing articles recognizing key contributions from the field of molecular cytogenetics, we are very pleased to launch our new journal blog to promote interaction among researchers. In this blog, we will engage in specific discussions with regards to new directions in the field and newly emerging technologies, as well as feedback from recent meetings/articles and more. The blog can be accessed at [20], and also through the journal homepage.

The field of molecular cytogenetics is now entering an exciting time. Following various large-scale sequencing projects that have revealed a high level of genetic heterogeneity, it is clear that single-cell resolution and cell population dynamics are essential to understanding the mechanisms of many common and complex diseases. Thus molecular cytogenetics has become increasingly important as it provides information on both individual cells and cell populations. We hope our journal Molecular Cytogenetics will promote the popularity of the molecular cytogenetic approach during the post-genomic era.

With the help of its readers and authors we look forward to the continuing growth of Molecular Cytogenetics.
C Biomed Central

(C) 2013 Liehr et al.; licensee BioMed Central Ltd. This is an Open Access article distributed under the terms of the Creative Commons Attribution License (http://creativecommons.org/licenses/by/2.0), which permits unrestricted use, distribution, and reproduction in any medium, provided the original work is properly cited. 
Table 1 Highly assessed/cited papers published in Molecular Cytogenetics (information valid for the end of June 2013)

\begin{tabular}{|c|c|c|c|c|c|}
\hline Reference & Title & Authors & Year/volume/number & Accesses & Citations \\
\hline [2] & On the origin of trisomy 21 Down syndrome & $\begin{array}{c}\text { Maj A Hultén, Suketu D Patel, Maira } \\
\text { Tankimanova, Magnus Westgren, Nikos } \\
\text { Papadogiannakis, Anna Jonsson, Erik Iwarsson }\end{array}$ & $\begin{array}{l}\text { Molecular Cytogenetics } \\
2008,1: 21\end{array}$ & 15036 & 56 \\
\hline [3] & $\begin{array}{l}\text { Human interphase chromosomes: a review of } \\
\text { available molecular cytogenetic technologies }\end{array}$ & $\begin{array}{c}\text { Svetlana G Vorsanova, Yuri B Yurov, } \\
\text { Ivan Y lourov }\end{array}$ & $\begin{array}{l}\text { Molecular Cytogenetics } \\
\text { 2010, 3:1 }\end{array}$ & 14502 & 35 \\
\hline [4] & $\begin{array}{l}\text { FISH mapping of Philadelphia negative } \\
\text { BCR/ABL1 positive CML }\end{array}$ & $\begin{array}{l}\text { Anna Virgili, Diana Brazma, Alistair G Reid, Julie } \\
\text { Howard-Reeves, Mikel Valgañón, Anastasios } \\
\text { Chanalaris, Valeria AS De Melo, David Marin, } \\
\text { Jane F Apperley, Colin Grace, Ellie P Nacheva }\end{array}$ & $\begin{array}{l}\text { Molecular Cytogenetics } \\
2008,1: 14\end{array}$ & 10908 & 19 \\
\hline [5] & $\begin{array}{l}\text { Selection of single blastocysts for fresh transfer } \\
\text { via standard morphology assessment alone and } \\
\text { with array CGH for good prognosis IVF patients: } \\
\text { results from a randomized pilot study }\end{array}$ & $\begin{array}{l}\text { Zhihong Yang, Jiaen Liu, Gary S Collins, } \\
\text { Shala A Salem, Xiaohong Liu, Sarah S Lyle, } \\
\text { Alison C Peck, E Sills, Rifaat D Salem }\end{array}$ & $\begin{array}{c}\text { Molecular Cytogenetics } \\
\text { 2012, 5:24 }\end{array}$ & 9981 & 33 \\
\hline [6] & $\begin{array}{c}\text { Mosaic 22q11.2 microdeletion syndrome: } \\
\text { diagnosis and clinical manifestations } \\
\text { of two cases }\end{array}$ & $\begin{array}{l}\text { Ashutosh Halder, Manish Jain, } \\
\text { Madhulika Kabra, Neerja Gupta }\end{array}$ & $\begin{array}{l}\text { Molecular Cytogenetics } \\
2008,1: 18\end{array}$ & 9728 & 17 \\
\hline [7] & $\begin{array}{l}\text { Fluorescence in situ hybridization in } \\
\text { combination with the comet assay and } \\
\text { micronucleus test in genetic toxicology }\end{array}$ & Galina G Hovhannisyan & $\begin{array}{c}\text { Molecular Cytogenetics } \\
\text { 2010, 3:17 }\end{array}$ & 8963 & 16 \\
\hline [8] & $\begin{array}{l}\text { The genome diversity and karyotype } \\
\text { evolution of mammals }\end{array}$ & $\begin{array}{l}\text { Alexander S Graphodatsky, } \\
\text { Vladimir A Trifonov, Roscoe Stanyon }\end{array}$ & $\begin{array}{c}\text { Molecular Cytogenetics } \\
2011,4: 22\end{array}$ & 8794 & 11 \\
\hline [9] & $\begin{array}{l}\text { Cytogenetic contribution to } \\
\text { uniparental disomy (UPD) }\end{array}$ & Thomas Liehr & $\begin{array}{c}\text { Molecular Cytogenetics } \\
\text { 2010, 3:8 }\end{array}$ & 8348 & 28 \\
\hline$[10]$ & $\begin{array}{l}\text { The hierarchically organized splitting } \\
\text { of chromosomal bands for all } \\
\text { human chromosomes }\end{array}$ & $\begin{array}{l}\text { Nadezda Kosyakova, Anja Weise, } \\
\text { Kristin Mrasek, Uwe Claussen, } \\
\text { Thomas Liehr, Heike Nelle }\end{array}$ & $\begin{array}{c}\text { Molecular Cytogenetics } \\
\text { 2009, 2:4 }\end{array}$ & 7881 & 5 \\
\hline [11] & $\begin{array}{l}\text { Autistic disorder associated with a paternally } \\
\text { derived unbalanced translocation leading } \\
\text { to duplication of chromosome } \\
\text { 15pter-q13.2: a case report }\end{array}$ & $\begin{array}{l}\text { David J Wu, Nicholas J Wang, } \\
\text { Jennette Driscoll, Naghmeh Dorrani, Dahai } \\
\text { Liu, Marian Sigman, N Carolyn Schanen }\end{array}$ & $\begin{array}{c}\text { Molecular Cytogenetics } \\
2009,2: 27\end{array}$ & 7801 & 6 \\
\hline
\end{tabular}

Table 2 Highly cited papers additional to those highly accessed

\begin{tabular}{|c|c|c|c|c|}
\hline Reference & Title & Authors & Year/volume/number & Citations \\
\hline [12] & $\begin{array}{l}\text { Expanding the clinical phenotype of the } 3 q 29 \\
\text { microdeletion syndrome and characterization } \\
\text { of the reciprocal microduplication }\end{array}$ & $\begin{array}{l}\text { Blake C Ballif, Aaron Theisen, Justine Coppinger, } \\
\text { Gordon C Gowans, Joseph H Hersh, Suneeta } \\
\text { Madan-Khetarpal, Karen R Schmidt, Raymond Tervo, } \\
\text { Luis F Escobar, Christopher A Friedrich, Marie } \\
\text { McDonald, Lindsey Campbell, Jeffrey E Ming, Elaine } \\
\text { H Zackai, Bassem A Bejjani, Lisa G Shaffer }\end{array}$ & $\begin{array}{l}\text { Molecular Cytogenetics } \\
\qquad 2008,1: 8\end{array}$ & 117 \\
\hline [13] & Chromosomal mosaicism goes global & Ivan Y lourov, Svetlana G Vorsanova, Yuri B Yurov & $\begin{array}{l}\text { Molecular Cytogenetics } \\
2008,1: 26\end{array}$ & 74 \\
\hline [14] & $\begin{array}{l}\text { Microdeletion of 6q16.1 encompassing EPHA7 } \\
\text { in a child with mild neurological abnormalities } \\
\text { and dysmorphic features: case report }\end{array}$ & $\begin{array}{l}\text { Traylor RN, Fan Z, Hudson B, Rosenfeld JA, } \\
\text { Shaffer LG, Torchia BS, Ballif BC }\end{array}$ & $\begin{array}{c}\text { Molecular Cytogenetics } \\
\text { 2009, 2:17 }\end{array}$ & 41 \\
\hline$[15]$ & $\begin{array}{l}\text { Comparative analysis of copy number } \\
\text { detection by whole-genome BAC } \\
\text { and oligonucleotide array CGH }\end{array}$ & $\begin{array}{l}\text { Neill NJ, Torchia BS, Bejjani BA, } \\
\text { Shaffer LG, Ballif BC }\end{array}$ & $\begin{array}{l}\text { Molecular Cytogenetics } \\
\text { 2010, 3:11 }\end{array}$ & 40 \\
\hline [16] & $\begin{array}{l}\text { Cryptic genomic imbalances in patients with } \\
\text { de novo or familial apparently balanced } \\
\text { translocations and abnormal phenotype }\end{array}$ & $\begin{array}{l}\text { Sismani C, Kitsiou-Tzeli S, loannides M, Christodoulou C, } \\
\text { Anastasiadou V, Stylianidou G, Papadopoulou E, } \\
\text { Kanavakis E, Kosmaidou-Aravidou Z, Patsalis PC }\end{array}$ & $\begin{array}{l}\text { Molecular Cytogenetics } \\
\text { 2008, 1:15 }\end{array}$ & 39 \\
\hline$[17]$ & $\begin{array}{l}\text { Chromosome distribution in human sperm - a } \\
\text { 3D multicolor banding-study }\end{array}$ & $\begin{array}{l}\text { Manvelyan M, Hunstig F, Bhatt S, Mrasek K, Pellestor F, } \\
\text { Weise A, Simonyan I, Aroutiounian R, Liehr T }\end{array}$ & $\begin{array}{l}\text { Molecular Cytogenetics } \\
\qquad 2008,1: 25\end{array}$ & 34 \\
\hline [18] & $\begin{array}{c}\text { GIN'n'CIN hypothesis of brain aging: deciphering } \\
\text { the role of somatic genetic instabilities and } \\
\text { neural aneuploidy during ontogeny }\end{array}$ & Yurov YB, Vorsanova SG, lourov IY & $\begin{array}{c}\text { Molecular Cytogenetics } \\
2009,2: 23\end{array}$ & 29 \\
\hline
\end{tabular}




\section{Acknowledgements}

We would like to express our appreciation to Mr. Sam Rose, our Journal Development Editor at BioMed Central, for his enthusiasm and efficiency in promoting the success of Molecular Cytogenetics.

\section{Author details}

'Jena University Hospital, Friedrich Schiller University, Institute of Human Genetics, Jena, Germany. ${ }^{2}$ Wayne State University School of Medicine, 540 E Canfield Street, Detroit, MI 48201, USA. ${ }^{3}$ National Research Center of Mental Health, Russian Academy of Medical Sciences, Moscow, Russia. ${ }^{4}$ Ameripath Florida, 8150 Chancellor Dr Ste 110, Orlando, FL 32809, USA.

Received: 18 July 2013 Accepted: 18 July 2013

Published: 24 July 2013

\section{References}

1. Liehr T, Heng H, Yurov YB: Reviewer acknowledgement 2013. Mol Cytogenet 2013, 6:9.

2. Hultén MA, Patel SD, Tankimanova M, Westgren M, Papadogiannakis N, Jonsson AM, Iwarsson E: On the origin of trisomy 21 down syndrome. Mol Cytogenet 2008, 1:21.

3. Vorsanova SG, Yurov YB, lourov IY: Human interphase chromosomes: a review of available molecular cytogenetic technologies. Mol Cytogenet 2010, 3:1.

4. Virgili A, Brazma D, Reid AG, Howard-Reeves J, Valgañón M, Chanalaris A, De Melo VA, Marin D, Apperley JF, Grace C, Nacheva EP: FISH mapping of Philadelphia negative BCR/ABL1 positive CML. Mol Cytogenet 2008, 1:14.

5. Yang Z, Liu J, Collins GS, Salem SA, Liu X, Lyle SS, Peck AC, Sills ES, Salem $\mathrm{RD}$ : Selection of single blastocysts for fresh transfer via standard morphology assessment alone and with array CGH for good prognosis IVF patients: results from a randomized pilot study. Mol Cytogenet 2012, $5: 24$.

6. Halder A, Jain M, Kabra M, Gupta N: Mosaic 22q11.2 Microdeletion syndrome: diagnosis and clinical manifestations of two cases. Mol Cytogenet 2008, 1:18

7. Hovhannisyan GG: Fluorescence in situ hybridization in combination with the comet assay and micronucleus test in genetic toxicology. Mol Cytogenet 2010, 3:17.

8. Graphodatsky AS, Trifonov VA, Stanyon R: The genome diversity and karyotype evolution of mammals. Mol Cytogenet 2011, 4:22.

9. Liehr T: Cytogenetic contribution to uniparental disomy (UPD). Mol Cytogenet 2010, 3:8.

10. Kosyakova N, Weise A, Mrasek K, Claussen U, Liehr T, Nelle H: The hierarchically organized splitting of chromosomal bands for all human chromosomes. Mol Cytogenet 2009, 2:4.

11. Wu DJ, Wang NJ, Driscoll J, Dorrani N, Liu D, Sigman M, Schanen NC: Autistic disorder associated with a paternally derived unbalanced translocation leading to duplication of chromosome 15pter-q13.2: a case report. Mol Cytogenet 2009, 2:27.

12. Ballif BC, Theisen A, Coppinger J, Gowans GC, Hersh JH, Madan-Khetarpal S, Schmidt KR, Tervo R, Escobar LF, Friedrich CA, McDonald M, Campbell L, Ming JE, Zackai EH, Bejjani BA, Shaffer LG: Expanding the clinical phenotype of the $3 q 29$ microdeletion syndrome and characterization of the reciprocal microduplication. Mol Cytogenet 2008, 1:8

13. Lourov IY, Vorsanova SG, Yurov YB: Chromosomal mosaicism goes global. Mol Cytogenet 2008, 1:26

14. Traylor RN, Fan Z, Hudson B, Rosenfeld JA, Shaffer LG, Torchia BS, Ballif BC: Microdeletion of $6 q 16.1$ encompassing EPHA7 in a child with mild neurological abnormalities and dysmorphic features: case report. $\mathrm{Mol}$ Cytogenet 2009, 2:17

15. Neill NJ, Torchia BS, Bejjani BA, Shaffer LG, Ballif BC: Comparative analysis of copy number detection by whole-genome BAC and oligonucleotide array CGH. Mol Cytogenet 2010, 3:11.

16. Sismani C, Kitsiou-Tzeli S, loannides M, Christodoulou C, Anastasiadou V, Stylianidou G, Papadopoulou E, Kanavakis E, Kosmaidou-Aravidou Z, Patsalis PC: Cryptic genomic imbalances in patients with de novo or familial apparently balanced translocations and abnormal phenotype. $\mathrm{Mol}$ Cytogenet 2008, 1:15

17. Manvelyan M, Hunstig F, Bhatt S, Mrasek K, Pellestor F, Weise A, Simonyan I, Aroutiounian R, Liehr T: Chromosome distribution in human sperm - a 3D multicolor banding-study. Mol Cytogenet 2008, 1:25.
18. Yurov YB, Vorsanova SG, lourov IY: GIN'n'CIN hypothesis of brain aging: deciphering the role of somatic genetic instabilities and neural aneuploidy during ontogeny. Mol Cytogenet 2009, 2:23.

19. Schlegelberger B: In memoriam: Prof. Dr. rer. nat. Dr. med. h.c. Lore Zech; 24.9.1923 - 13.3.2013: honorary member of the European society of human genetics, honorary member of the German society of human genetics, doctor laureate, the University of Kiel, Germany. Mol Cytogenet 2013, 6:20

20. Molecular cytogenetics blog. http://blogs.biomedcentral.com/molcyt.

doi:10.1186/1755-8166-6-28

Cite this article as: Liehr et al:: Molecular Cytogenetics: the first impact factor (2.36). Molecular Cytogenetics 2013 6:28.

\section{Submit your next manuscript to BioMed Central and take full advantage of:}

- Convenient online submission

- Thorough peer review

- No space constraints or color figure charges

- Immediate publication on acceptance

- Inclusion in PubMed, CAS, Scopus and Google Scholar

- Research which is freely available for redistribution 